\title{
Descripción del Somatotipo e IMC en una Muestra de Adolescentes de Colegios Municipalizados de la Ciudad de Temuco - Chile
}

\author{
Description of Somatotype and BMI the Adolescent's Sample to Public School of the Temuco-Chile \\ "Cristián Martínez; **Héctor Silva; **Erika Collipal \& *Vanessa Carrasco S.
}

MARTÍNEZ, C.; SILVA, H.; COLLIPAL, E. \& CARRASCO, V. Descripción del somatotipo e IMC en una muestra de Adolescentes de colegios municipalizados de la ciudad de Temuco - Chile. Int. J. Morphol., 26(3):653-657, 2008.

RESUMEN: La mal nutrición en el mundo se ha convertido en un problema de salud pública, similar situación ocurre en Chile. Los estudios del índice de masa corporal (IMC), de la composición corporal, estado nutricional, de la población, son los parámetros recomendados por la OMS (Organización Mundial de la Salud), para conocer el estado nutricional. Realizamos este estudio para describir la realidad nutricional de los adolescentes de los colegios municipalizados de la ciudad de Temuco. Evaluamos 993 adolescentes de ambos sexos, 543 hombres $(54,7 \%)$ y 450 mujeres (45,3\%), entre 6 y 14 años de edad, alumnos de diferentes colegios urbanos municipalizados. Para obtener el somatotipo utilizamos el método antropométrico descrito por Heath \& Carter, efectuándose la evaluación y clasificación del IMC de acuerdo a las normas de la OMS. Los hombres, fueron más mesomórficos que las mujeres, siendo esta diferencia estadísticamente significativa. $\mathrm{p}<=0,05$. Al comparar los componentes Ectomorfía y Mesomorfía del somatotipo en relación al sexo presentaron valores estadísticamente significativos a favor de los varones, pero el valor de Endomorfía fue estadísticamente significativo a favor de las mujeres. El 19,4 \% del total de la muestra eran obesos, y un 23,1\% presentaron sobrepeso. En los hombres el 19,3\% fueron obesos y un 23,9\% presentaron sobrepeso, en las mujeres el 22,0 \% sobrepeso y un $19.6 \%$ obesidad. Observamos un predominio del componente endomesomórfico en las mujeres y mesoendomórficos en los varones. Los probables factores son la modificación de estilo de vida, alimentación y efecto del sedentarismo que en la Novena Región alcanza un $96,4 \%$ de la población.

PALABRAS CLAVE: Somatotipo; Antropometría; Índice de masa corporal.

\section{INTRODUCCIÓN}

La obesidad es una enfermedad que afecta a la mayoría de la población América Latina, y que ha llevado a la Organización Mundial de la Salud a postular que es una epidemia global.

En Chile, la obesidad constituye el problema nutricional más relevante de la salud de la población en todas las edades. Escolares de 6 años que ingresaron a primer año básico en 2004, presentaron una prevalencia de obesidad de 17,3\% (peso/talla $>2$ de NCHS), mientras que en los preescolares asistentes a jardines de la Junta Nacional de Jardines Infantiles (JUNJI), esta cifra fue de 10,6\% en 2005. (JUNAEB, 2004; JUNJI, 2005)

La Encuesta Nacional del Deporte de 1996, en una muestra de 4300 personas entre 10 y 70 años, reveló que el $83 \%$ de los individuos eran sedentarios, la que afectaba con mayor intensidad a mujeres de nivel socioeconómico bajo, siendo un factor que incide en el alto porcentaje de obesidad en todos los grupos etarios de nuestra población.

Según estudios y encuestas realizadas por el Ministero de Salud (MINSAL), existe un 33\% de sobrepeso y obesidad en escolares púberes de la IX Región, Chile y que en la encuesta nacional de salud 2003 del SNS se encontró que la obesidad afecta al $22 \%$ de las personas, con un aumento significativo a través de los grupos de edad, subiendo de $9 \%$ a $29 \%$ entre el grupo de menores de 25 años y el de los mayores de 64 años. Las mujeres tienen significativamente mayor obesidad (25\%) que los hombres (19\%), a pesar que éstos tenían mayor sobrepeso (MINSAL, 2003)

Los estudios de obesidad y del IMC han sido escasos en la IX Región de Chile. Según el Ministerio de Salud de

\footnotetext{
* Dpto. de Educación Física, Facultad de Educación y Humanidades, Universidad de La Frontera, Chile.

**Dpto. de Ciencias Básicas, Facultad de Medicina, Universidad de La Frontera, Chile.

Proyecto Diufro No 110.602 . Dirección de Investigación y Desarrollo de la Universidad de La Frontera, Chile.
} 
Chile, existe un $25 \%$ de sobrepeso y obesidad en menores de 6 años, controlados en el Sistema Nacional de Servicios de Salud, un $35 \%$ en preescolares que asisten a Jardines Infantiles del Gobierno, un 38\% en escolares de 1er año Básico, un $33 \%$ en escolares púberes y cerca de un $50 \%$ en adultos, dependiendo de la edad y sexo. Por otro lado, 40 a $76 \%$ de los escolares obesos serán adultos obesos. En un estudio de prevalencia realizado en Santiago mostró que la epidemia de obesidad en Chile está afectando en forma progresiva a los preescolares de menor edad, dándose la paradoja, que junto con estar concluyendo la celebración de la erradicación de la desnutrición, se debe afrontar las consecuencias de la obesidad (Kain et al., 2007).

Desde este punto de vista la antropometría tiene como finalidad conocer las característica morfológicas de una muestra, para poder visualizar esas características se usa la del técnica del somatotipo. Esta usa los componentes Endomórfico, Mesomórficos y Ectomórfico. El concepto vigente del Somatotipo fue propuesto por Heath y Carter, quienes se apoyaron en los conceptos teóricos de Sheldon (Carter \& Heath, 1990).

La escala del somatotipo se puede caracterizar por medio de valores que son cualitativos y cuantitativos, se pueden denominar como bajo, moderado, elevado y extremadamente alto, o valores que van desde 0,1 a 7 .

El análisis del somatotipo ha sido realizado en poblaciones normales de diferentes edades, sexo y niveles socioeconómicos para conocer las características biotipológicas de estos grupos humanos (Katzmarzyk et al., 1998; Katzmarzyk \& Malina, 1999).

Valores específicos de sus componentes han sido correlacionados en diferentes patologías, cáncer de mama, cardiopatías, escoliosis y obesidad (Magnusson et al., 1998) Además, en el deporte permite conocer el estado físico de una población deportiva y el estado nutricional de los individuos, también comparar entre especialidades, genero y señalar las tendencias (Valkov et al., 1996; Carter \& Ackland, 1998) y realizar la búsqueda de talentos deportivos y modificar los componentes, mejorando el rendimiento según el deporte que se practique (Toro et al., 1983; Almagià et al., 1996).

El análisis de los pliegues cutáneos permite estimar el contenido de masa grasa y masa libre de grasa en los diferentes individuos y, consecuentemente, inferir los requerimientos nutricionales. (Toro \& Almagià, 1989; Depress, 1997; Swan \& McConnell, 1999).

En Chile, se ha encontrado gran variedad de somatotipos individuales, que se distribuyen en los sectores endomesomórficos y mesoendomórficos. Silva et al. (2003) observó un predominio del componente endomesomórfico en una muestra de adolescentes de la IX Región, Chile, lo que indica la existencia de un alto componente de tejido adiposo, concordante con el aumento de peso y con la obesidad en los adolescentes.

El propósito del presente estudio fue describir el somatotipo e IMC de una población de adolescentes de colegios municipalizados de la IX Región, Chile.

\section{MATERIAL Y MÉTODO}

Para el estudio del somatotipo y del IMC, fueron evaluados 993 hombres 543 hombres $(54,7 \%)$ y 450 mujeres $(45,3 \%)$ cuyas edades fluctuaron entre los 6 y 14 años, provenientes de colegios municipales de la ciudad de Temuco, IX Región, Chile.

Los sujetos fueron evaluados en sus colegios. Los datos obtenidos fueron registrados en una ficha de tres hojas idénticas autocopiativas, lápiz dermográfico, balanza Detecto, banco de antropometría, estadíometro de pared, adipómetro Harpenden, antropómetro Lafayette y cinta antropométrica de metal Sany.

Para la obtención del somatotipo utilizamos el método antropométrico de Heath and Carter, que incluye las variables pliegues cutáneos, perímetros, diámetros, y además, peso, talla y edad. Para la clasificación de los somatotipos, las mediciones fueron registradas en una ficha antropométrica computacional.

El IMC se calculó a través de la formula (P/T2), que, según la OMS, los individuos son clasificados: en enflaquecidos, normal, sobrepeso y obeso, diferenciados por edad y sexo.

Para el procesamiento y análisis de los datos se utilizó el programa estadístico SPSS 11.0.

\section{RESULTADOS}

La clasificación del somatotipo e índice de masa corporal en adolescentes de ambos sexos según edad en TemucoChile y los componentes del somatotipo en la misma muestra, pueden ser observados en las Tablas I, II y III. En la Tabla IV se expresan los valores del IMC de ambos sexos. 
MARTíneZ, C.; SILVA, H.; COLLIPAL, E. \& CARRASCO, V. Descripción del somatotipo e IMC en una muestra de Adolescentes de colegios municipalizados de la ciudad de Temuco - Chile. Int. J. Morphol., 26(3):653-657, 2008.

Tabla I. Clasificación del somatotipo e índice de masa corporal en adolescentes de ambos sexos según edad en Temuco-Chile. $n=996$

\begin{tabular}{crcccc}
\hline Edad & \multicolumn{1}{c}{$\mathrm{n}$} & Endomorfía & Mesomorfía & Ectomorfía & IMC \\
\hline 6 & 37 & 4,3 & 4,7 & 1,6 & 17 \\
7 & 124 & 4,8 & 5,0 & 1,3 & 20,5 \\
8 & 79 & 4,6 & 4,6 & 1,8 & 19 \\
9 & 91 & 4,9 & 5,1 & 1,6 & 20 \\
10 & 170 & 5,7 & 4,9 & 1,7 & 29 \\
11 & 163 & 5,4 & 2,0 & 2,1 & 27 \\
12 & 113 & 4,8 & 4,3 & 2,0 & 21 \\
13 & 118 & 5,1 & 4,7 & 2,1 & 22 \\
14 & 101 & 5,2 & 4,5 & 1,9 & 23 \\
\hline
\end{tabular}

Tabla II. Clasificación del somatotipo e índice de masa corporal, en adolescentes dividido por sexos y según edad en Temuco-Chile $\mathrm{n}=996$

\begin{tabular}{cccccccccc}
\hline Edad & \multicolumn{2}{c}{$\mathrm{n}$} & \multicolumn{2}{c}{ Endomorfía } & \multicolumn{2}{c}{ Mesomorfía } & \multicolumn{2}{c}{ Ectomorfía } & IMC \\
\hline \multicolumn{1}{r}{} & Hombres / Mujeres & \multicolumn{2}{c}{ Hombres / Mujeres } & Hombres / Mujeres & Hombres / Mujeres \\
\hline 6 & 14 & 23 & 3,6 & 4,7 & 5,1 & 4,5 & 1,7 & 1,5 & 17 \\
7 & 69 & 55 & 4,5 & 5,2 & 5,1 & 4,8 & 1,4 & 1,2 & 21 \\
8 & 48 & 31 & 4,3 & 5,1 & 4,7 & 4,4 & 1,8 & 1,5 & 19 \\
9 & 54 & 37 & 4,4 & 5,6 & 5,2 & 4,9 & 1,1 & 1,9 & 20 \\
10 & 90 & 80 & 4,8 & 6,6 & 5,1 & 4,6 & 1,7 & 1,7 & 29 \\
11 & 90 & 73 & 5,3 & 5,4 & 4,7 & 3,9 & 1,4 & 1,8 & 27 \\
12 & 68 & 55 & 4,6 & 5,2 & 4,6 & 3,9 & 2,0 & 1,9 & 21 \\
13 & 62 & 56 & 4,3 & 5,4 & 5,5 & 3,9 & 2,3 & 1,7 & 22 \\
14 & 49 & 52 & 4,0 & 6,3 & 4,6 & 4,3 & 2,4 & 1,4 & 23 \\
\hline
\end{tabular}

Tabla III. Componentes del somatotipo en una muestra de adolescentes de ambos sexos de la ciudad de Temuco - Chile $\mathrm{n}=996$.

\begin{tabular}{lcc}
\hline & Hombres & Mujeres \\
\hline & $\mathrm{X}$ & $\mathrm{X}$ \\
Endomorfía & 4,6 & 5,7 \\
Mesomorfía & 5,0 & 4,3 \\
Ectomorfía & 1,9 & 1,7 \\
\hline
\end{tabular}

Tabla IV. Valores del IMC de ambos sexos en una muestra de adolescentes de la ciudad de Temuco-Chile $\mathrm{n}=996$.

\begin{tabular}{lcccccc}
\hline & \multicolumn{2}{c}{ Hombres } & \multicolumn{2}{c}{ Mujeres } & \multicolumn{2}{c}{ Total } \\
\hline Obeso & $\mathrm{n}$ & $\%$ & $\mathrm{n}$ & $\%$ & $\mathrm{n}$ & $\%$ \\
Sobrepeso & 105 & 19,3 & 88 & 19,6 & 193 & 19,4 \\
Normal & 130 & 23,1 & 99 & 22,0 & 229 & 23,1 \\
Enflaquecido & 267 & 49,2 & 227 & 50,4 & 494 & 49,8 \\
\hline
\end{tabular}

La prueba de T Student para muestras independientes en relación al sexo determinó que existen diferencias significativas en los componentes de ectomorfía mesomorfía, endomorfía. 


\section{DISCUSIÓN}

En Chile, las condiciones de salud han cambiado, lo que se ha traducido en una disminución en los índices de mortalidad y desnutrición. Varios autores concuerdan que una nutrición adecuada en las etapas tempranas de la vida, existiría una menor probabilidad de contraer enfermedades, ya sea en la niñez como en la edad adulta. Así, una alimentación balanceada se traducirá en un mejor desarrollo cerebral, en aumentos del crecimiento corporal, de la masa y de la composición muscular. En etapas más tardías se podrá destacar una mejor capacidad cognitiva, mayor rendimiento escolar, aumento de los parámetros inmunológicos, mayor capacidad para el trabajo físico y disminución del desarrollo de enfermedades crónicas no transmisibles, tales como la hipertensión arterial, cáncer, accidentes vasculares y envejecimiento (Uauy \& Castillo, 2001; Urrutia et al., 2001; Amigo et al., 2002).

La asociación entre mayor peso corporal y menarquia temprana también conlleva riesgos metabólicos a largo plazo. Estudios en adultos han determinado que a menor edad de la menarquia existe un mayor riesgo de insulino resistencia, dislipidemia, sobrepeso y aumento de la adiposidad abdominal, mayores cifras de presión arterial y mayor frecuencia de intolerancia a la glucosa (Remsberg et al., 2005; Kindblom et al., 2006).

En Chile, los estudios sobre obesidad señalan que es de alta incidencia en la población, según (JUNAEB; JUNJI) esta epidemia afecta a la mayoría de las personas iniciándose en los primeras etapas de la vida, que determinarían el tipo de somatotipo y además la predisposición a padecer enfermedades crónicas no transmisibles (Uauy \& Castillo; Urrutia et al.; Amigo et al.).

En nuestro estudio realizado en la población escolar municipalizada de la ciudad de Temuco la prevalencia de la obesidad fue de 19,3\% en los varones y 19,6 en las mujeres lo que concuerda con la encuesta nacional de salud MINSAL 2003. Al relacionar la obesidad y el sobrepeso se encontró que los varones tenían un $42,4 \%$ y de $41,6 \%$ en mujeres, lo que difiere con los resultados obtenidos en la encuesta realizada por el Minsal, en la cual encontró que existe un 33\% de sobrepeso y obesidad en escolares de la Novena región, lo que significa que la obesidad a aumentado con los años. Los factores determinantes en el aumento de esta enfermedad son el sedentarismo con un 96,4\% en la novena región (Chiledeportes, 2007) y los cambios en los estilos de vida de la población.

Dada la alta prevalencia de sobrepeso y obesidad en escolares y adolescentes en Chile, nos parece necesario implementar la evaluación periódica de este grupo etario, el que tradicionalmente no se controla regularmente. Esto implica que se implementen en los colegios normas nutricionales y una constante evaluación en los diferentes grupos etarios que conforman la población escolar.

MARTíneZ, C.; SILVA, H.; COLLIPAL, E. \& CARRASCO, V. Description of somatotype and BMI the adolescent's sample to public school of the Temuco- Chile. Int. J. Morphol., 26(x):653-657, 2008.

SUMMARY: Bad nutrition has become a public health problem, similar situation had happened in Chile studies on body composition, nutritional state, and body mass index (BMI), are parameters recommended by the WHO for determining human beings' nutritional state. This study has been carried out to describe the adolescent nutritional situation of the urban public schools in Temuco - Chile. Nine thousands and ninety nine subjects were evaluated, 543 men (54.7\%) and 450 women (46.3\%), between 6 and 14 years old, all students at urban public schools. In order to obtain the Somatotype the anthropometric Heath \& Carter method was used, classifying BMI according to the WHO norms. Men were more endomorphic than women, this difference was statistically significant $\quad(\mathrm{p}=0.005)$. To compare the ectomorphic and mesomorphic components of somatotopic in relation to sex, this values were statistic statistically significant to men, but the endomorphic value was statistically significant was to women. Regarding to the total sample, $19.4 \%$ was obese and $23.1 \%$ was overweight. Then, according to men, $19.3 \%$ were obese and a $23.9 \%$ were overweight, and women had a $22.0 \%$ of overweight and a $19.6 \%$ were obese. It was observed a prevalence of the endomesomorphic component in women and mesoendomorphic in men. These results show an obesity prevalence of $19.4 \%$ and an overweight of $23.1 \%$ within the evaluated population getting closer to the national standards of the adult population, which corresponds to a $50 \%$ according to the a survey given by the Chilean Ministry of Health (MINSAL), the probable factors are the modification of lifestyle, food and sedentary effect that in the Chilean ninth region reaches a $96.4 \%$ out of the population.

KEY WORDS: Somatotipe; Antropometry; Body mass index.

\section{REFERENCIAS BIBLIOGRÁFICAS}

Almagià, F. A.; Toro, D. T.; Binvignat, G. O.; Cabrera, E. \& Marinao, A. Aproximación al perfil morfoestructural y dismorfismo sexual de jóvenes de ambos sexos de edad de 6 a 23 años de edad caracterizados por el somatotipo.

Rev. Chil. Anat., 14(2):189-97, 1996. 
Amigo, H.; Bustos, P. \& Zumelzu, E. Cambio secular de las variables antropométricas al nacer: una perspectiva local. Rev. Med. Chile, 130:1095-100, 2002.

Carter J. \& Heath, H. Somatotyping: develoment and applications. $1^{\circ}$ Ed. New York, Cambridge University Press, 1990.

Carter J. \& Ackland, T. Sexual dimorphism in the physiques of Wold Championship divers. J. Sport Sci., 16:317-29, 1998.

Chiledeportes. Encuesta nacional de hábitos de actividad física y deporte en la población chilena igual o mayor a 18 años. Chile 2007. Disponible en: http:// w w w. chile de porte s.gov.cl/pdfs/ Documento\%20metodologico.pdf.

Depress, P. Visceral obesity, insulin resistance and dyslipidemia: contribution of endurance exercise training to the treatment of the plurimetabolic syndrome. Exercise and Sport Scienc., 25:271-300, 1997.

JUNAEB (Junta Nacional de Auxilio Escolar y Becas de Chile). Disponible en: http://www.junaeb.cl. Chile, 2004.

JUNJI (Junta Nacional de Jardines Infantiles de Chile). Disponible en: http://www.junji.cl. Chile, 2005.

Kain, J.; Lera, L.; Rojas, J. \& Uauy, R. Obesidad en preescolares de la Región Metropolitana de Chile. Rev. méd. Chile, 135(1):63-70. 2007.

Katzmarzyk, P.; Malina, R.; Song, T.; Theriault, G. \& Bouchard, C. Physique and echocardiographic dimensions in children, adolescent and young adults. Ann. Hum. Biol., 25:145-57, 1998.

Katzmarzyk, P. \& Malina, R. Body size and Physique among Canadians of first nation and Europena ancestry. Am. J. Phys. Anthrpol., 108:161-72, 1999.

Kindblom, J. M.; Lorentzon, M.; Norjavaara, E.; Lonn, L.; Brandberg. J. \& Angelhed, J. E.; Hellqvist, A.; Nilsson, S. \& Ohlsson, C. Pubertal timing is an independent predictor of central adiposity in young adult males: the Gothenburg osteoporosis and obesity determinants study. Diabetes, 55: 3047-52. 2006.

Magnusson, C.; Baron, J.; Persson, I.; Wolk, A.; Bergstrom, R.; Trichopoulos, D. \& Adami, H. O. Body size in different periods of life and breast cancer risk in postmenopausal women. Int. J. Cancer, 76:29-34, 1998.
MINSAL (Ministerio de Salud, Chile). Primera encuesta nacional de salud Chile 2003. Disponible en: http:// epi.minsal.cl/epi/html/invest/ENS/ENSmayo2004.pdf

Remsberg, K. E.; Demerath, E. W.; Schubert, C. M.; Chumlea, W. C.; Sun, S. S. \& Siervogel, R. M. Early menarche and the development of cardiovascular disease risk factors in adolescent girls. J. Clin. Endocrinol. Metab., 90:2718-24. 2005.

Uauy, R. \& Castillo, C. Nutrición de los niños en Chile: donde estamos, hacia donde vamos. Rev. Chil. Pediatr., 72 (1):15,2001

Urrutia, S.; Mardones, F. \& Salazar, G. Asociación entre la composición corporal de la embarazada y la composición corporal del recién nacido Rev. Ch. Pediatr., 72 (3):212-8, 2001.

Toro, T.; Arenas G. \& Almagià, A. Caracterización somatotípica de jóvenes estudiantes de Valparaíso. Anal. Anat. Normal, 1:101-6, 1983.

Toro, T. \& Almagià, A. Aplicación de estándares antropométricos para la evaluación del crecimiento y estado nutricional en niñas y niños de 11-15 años de Valparaíso. An. Anat. Nor., 7:99-107, 1989.

Silva, H.; Bruneau, J. C.; Reyno, H. P. \& Bucarey, S. Somatotipo e índice de masa corporal en una muestra de adolescentes de ambos sexos de la ciudad de Temuco, Chile. Int. J. Morphol., 21(4):309-13. 2003.

Swan, P. \& McConnell, K. Anthropometry and bioelectrical impedance inconsistently predicts fatness in women with regional adiposity. Med. Scienc. Sport. Exerc., 31(7):1068-75, 1999.

Valkov, J.; Matev, T. \& Hristov, I. Relation between somatype and some risk factors for ischemic heart disease. Folia Med. Plovdiv., 38:17-21, 1996.

\section{Dirección para Correspondencia: \\ Prof. Héctor Silva Mella \\ Facultad de Medicina \\ Universidad de La Frontera \\ Casilla 54-D \\ Temuco - CHILE}

Email:hsilva@ufro.cl

Recibido : 29-06-2008

Aceptado: 08-08-2008 
